\title{
Des lectures pour tous : les « bouquins » de Béatrice Poncelet
}

Singuliers albums que ceux de Béatrice Poncelet, aux deux sens de l'adjectif : ils peuvent paraître étranges, atypiques, et, dans le même temps, ils composent une œuvre unique, originale, que l'on reconnaît d'emblée, alors même qu'elle fait appel à toutes les techniques, au point d'afficher, selon le mot de Sophie Van Der Linden, un «non style ${ }^{1} »$. Des albums «hors cadre $\left(\mathrm{s}^{2}\right) »$, qui déconcertent, voire découragent celui qui se contente d'un rapide feuilletage, s'interroge sur leur destinataire et les juge d'accès trop difficile pour les enfants. Ces albums inclassables ne présentent pas de personnages identifiables et ne racontent pas vraiment d'histoires. On voit des objets, des décors. On entend des voix, jamais référencées. Texte et image se lient dans un entrelacs aux mailles serrées qui correspond à la définition de l'iconotexte, par Alain Montandon, comme «genre » essentiellement dialogique, qui se tient à l'écart de « ses deux bords externes que sont la légende et l'illustration ${ }^{3}$ ». De par sa formation, Béatrice Poncelet apparaît avant tout comme artiste ${ }^{4}$ et graphiste ; lorsqu'elle compose un ouvrage, le travail sur les images est premier, mais, dans l'album achevé, le texte s'avère indispensable : sans lui, elles ne sont pas lisibles, de même que, sans images, le texte resterait lettre morte. Ecrit par bribes, et progressivement intégré à l'ensemble plastique et graphique, il n'est pas pour autant subalterne : son emplacement est réservé, inclus dans l'image, à laquelle, par delà les fonctions d'ancrage et de relais définies par Barthes, il sert de révélateur ; de plus, il comporte lui-même, par les choix de mise en page et de typographie, une forte part d'iconicité.

Pour évoquer la singularité de ce que Poncelet elle-même appelle, de façon un peu expéditive, ses «bouquins », je m'attacherai successivement aux voix entendues et aux objets montrés, avant de souligner comment, alors que la complexité de l'œuvre captive le lecteur adulte, Poncelet se pose toujours la question du jeune lecteur, et justifie son inscription dans le champ de la littérature de jeunesse. Il s'agit, en somme, de montrer en quoi ces albums sont des lectures pour tous.

\footnotetext{
${ }^{1}$ S.Van der Linden, , Lire l'album, L'atelier du poisson soluble, 2006, p. 144.

${ }^{2}$ Pour reprendre le titre de l'Université d'été de l'Institut International Charles Perrault (2007), où B. Poncelet était, à ce juste titre, invitée.

${ }^{3}$ A. Montandon (dir.), Iconotextes, CRCD, Ophrys, 1990, p. 5. L'auteur reprend le terme forgé par M. Nehrlich.

${ }^{4}$ Même si elle récuse ce terme.
} 


\section{Des voix et des objets}

\section{Voix anonymes}

A l'exception du premier, qu'elle renie, tout album de Poncelet est écrit à la première personne, en accord avec un choix énonciatif prépondérant dans la littérature de jeunesse contemporaine. On entend parfois ce je dès le titre : Je reviendrai le dimanche 39, Je pars à la guerre, je serai là pour le goûter, Je le loup et moi. Seul des 12 albums publiés en 25 ans, T'aurais tombé comporte également un texte que l'on pourrait qualifier de didascalique, quelques fragments à la troisième personne, distingués par la typographie.

Ce narrateur peut être un adulte, mais il s'agit le plus souvent d'un enfant, terme à prendre dans une acception large, fille ou garçon, plutôt une grande fille, à l'orée de l'adolescence, ou un petit garçon. Ce qui frappe lorsqu'on écoute les voix qui s'expriment dans ces ouvrages, c'est qu'aucun idiolecte ne les distingue : on n'en reconnaît qu'un, celui de l'auteur elle-même. Ces voix s'adressent parfois à un destinataire intradiégétique, dans un dialogue entre enfant et adulte, dialogue tendre dans T'aurais tombé, où une mère et son petit garçon évoquent, au moment du coucher, le souvenir d'un accident traumatisant, ou dialogue de sourdes, lors du petit déjeuner, entre une adolescente et sa mère, dans Et la gelée, framboise ou cassis?. Dans Semer, la voix qui s'exprime au fil des pages est celle d'une mère qui parle à son enfant.

Mais je vois que je joue malgré moi mon rôle de lecteur en précisant les liens familiaux que je suis conduite à inférer à partir des voix entendues. En réalité, jamais les mots «mère », «fille », «fils » ne sont écrits dans Et la gelée, ou dans Semer. Après T'aurais tombé, où elle précise encore «le père, la mère, l'enfant», Poncelet gomme peu à peu ces indications ; dans Chut! elle lit, elle évoque encore, dans la bouche de la narratrice «nos parents », «ma sœur », «l'autre frère », pour introduire les personnages, puis elle se contente de pronoms sans référents ou de substituts nominaux. A partir de Chez Elle, où un enfant évoque les adultes auxquels on le confie sans jamais préciser de lien familial entre eux, Poncelet recherche la plus grande généralité possible et tâche de reculer le moment où l'accord d'un participe ou d'un adjectif révèlera le sexe du narrateur: il faut attendre la dernière page pour apprendre, avec la phrase «quand je serai grande », que la voix entendue était celle d'une petite fille; c'est seulement à la septième double page que le narrateur de Chaise et café dit qu'il est caché dans l'ombre pour épier celui où le lecteur entrevoit peutêtre un grand frère. 
Tous ces albums sont des albums de famille. De l'un à l'autre, se tissent inlassablement des relations intergénérationnelles, et l'on pourrait dire que tous les livres de Poncelet n'en font qu'un, pour composer une œuvre particulièrement homogène, qui explore les liens familiaux, dans tous les sens, à partir de tous les points de vue : dans la fratrie, entre parents et enfants, enfants et adultes, entre les adultes et leurs aînés. Elle parle au nom de l'expérience intime et introduit son lecteur dans l'espace familial, au cœur du foyer, pour pointer émotions et sentiments liés aux événements du quotidien, où chacun peut retrouver, à travers de menus drames et des trames familières, les échos de sa propre vie.

\section{Poncelet, lectrice de Sarraute}

Poncelet ne fait pas mystère de son goût pour certains des auteurs du Nouveau Roman, surtout Nathalie Sarraute; comment ne pas reconnaître dans quelques-unes des caractéristiques soulignées ci-dessus cette influence omniprésente sur ses textes mais aussi sur ses images?

Les rapprochements entre deux attitudes radicales sont évidents, et, au premier chef, le renoncement à la représentation du personnage, dont Sarraute signe la condamnation à mort en 1956, dans L'ère du soupçon. Chez Poncelet, il n'est jamais nommé, ni décrit, et n'apparaît pas davantage sur les images ; anonymé, neutre, parfois asexué, après gommage de sa part historique, sociale, individuelle, il peut tendre vers l'universalité ; il devient une voix, simple support de la parole, et, comme chez Sarraute, cette voix est adressée. Les phrases de Dominique Rabaté sur Sarraute pourraient convenir au travail de Poncelet :

Le discours n'est jamais monologue : il va vers l'autre, réclame sa présence et sa complicité. La voix narratrice ne peut pas plus se passer de cet effet d'adresse constant. En ce sens, c'est bien la parole et son usage qui sont au premier plan ${ }^{5}$.

Plusieurs stratégies sont ainsi mobilisées pour [...] conserver mobilité et vibrato [à la parole], à commencer par l'usage - si souvent commenté - des pointillés, de la phrase suspendue, mais aussi des interrogations ${ }^{6}$.

Chez Poncelet, les incises des verbes introducteurs sont absentes, les ouvrages débutent in medias res par un discours, des points de suspension, parfois sans majuscules, les titres sont du même type. Questions sans réponses, ouvertures et finales suspensives ou interrogatives, inscrivent les textes - mais aussi les images - de ces albums dans une «esthétique de

\footnotetext{
${ }^{5}$ D. Rabaté, Le Chaudron fêlé, Paris, José Corti, 2006, p. 91.

${ }^{6}$ Ibid., p. 95.
} 
l'incomplétude ${ }^{7}$ », où rétention, non-dit, fragments procurent une impression d'inachèvement et ouvrent un espace de choix à l'activité du lecteur.

Poncelet joue parfois avec son modèle, sur le mode parodique : la phrase de clôture de Galipette, «Vous les entendez ? », est le titre d'un texte de Sarraute où s'opposent également univers enfant et adulte. Mais Poncelet la place dans la bouche des enfants. De même, on peut voir un pastiche de certaines conversations mondaines lues chez Sarraute lorsque les phrases de l'adulte futile de Chez Elle se dévident en boucle dans un texte tronqué, peu lisible, blanc sur jaune, afin de traduire plastiquement le propos superficiel d'une parole qui tourne à vide.

Sarraute déclare qu'elle tente « de communiquer au lecteur [...] par des images qui en donnent des équivalents et lui fassent éprouver des sensations analogues » les «véritables

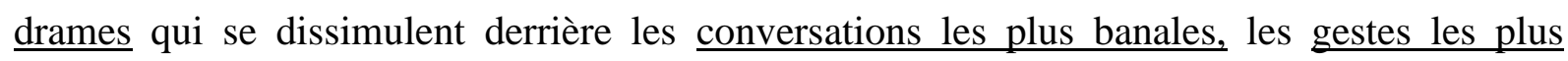
quotidiens $^{8} »$. De même, Poncelet prête attention aux mouvements ténus de la conscience, dans un style fortement marqué par l'oralité, et la dimension iconotextuelle de son travail lui permet de rendre visibles ces sentiments profonds. Ainsi, dans Et la gelée..., elle fait entendre une «sous-conversation », en nous introduisant dans le flux de conscience d'une adolescente qui, lors d'une scène triviale, le petit déjeuner en famille, se découvre un visage ridé dans le liquide refroidi au fond de son bol, et prend soudain conscience du caractère éphémère de la jeunesse, ce que traduit l'image par une galerie de portraits, d'œuvres d'art et de représentations symboliques du passage du temps.

\section{Objets inanimés?}

La notion de personnage est également déconstruite dans l'image, où se succèdent décors vides et superpositions d'objets. Nuançons toutefois: on aperçoit bien quelques silhouettes, mais de dos, très stylisées, ou tronquées, donc anonymées, comme cet inconnu rencontré dans le bus, dans Je, le loup et moi, successivement homme tronc, silhouette noire, ou objet lorsque le texte révèle l'image : « Il me faisait penser à mon modèle d'anatomie ».

\subsection{Photographies}

On voit bien quelques visages, mais ils renvoient toujours à des objets : masques, têtes de poupées, dans Fée ? et Galipette, reproductions de tableaux, dans Chez Elle ou Et la gelée. Les rares figures humaines entrevues, également réifiées, apparaissent sur des portraits photographiques retravaillés, déformés, barbouillés, découpés. Dans T'aurais tombé, des

\footnotetext{
${ }^{7}$ D. Rabaté, Poétiques de la voix, Paris, José Corti, 1999, p.164.

${ }^{8}$ N. Sarraute, Préface à L'ère du soupçon, Idées, NRF, Gallimard, 1956, p. 8-9, c'est moi qui souligne.
} 
photographies de fragments de mains ou de visages s'insèrent dans les compositions d'objets qui représentent les lieux traversés : la maison, l'hôpital. Les mains de l'adulte et de l'enfant encadrent le livre lu au moment du coucher; la nuit d'hôpital est traduite par une image composite sur une double page sans texte : sur la belle page, à droite, soudé dans l'agencement de trois photographies détourées, le trio familial dort, tandis que l'heure tourne - matérialisée par une horloge -, sous le regard d'un étrange vigile aux yeux écarquillés, la radiographie du crâne de l'enfant que le regard du lecteur découvre in fine page de gauche. Tout est dit sans un mot, la fusion affective et l'angoisse qui rôde.

Dans Je le loup et moi, ainsi que dans Et la gelée, une seule photo, toujours la même, mais disposée et colorée de différentes façons, indique visuellement la narratrice. Dans Chut! elle lit, certains personnages apparaissent sur des photographies qui signalent la présence latente d'une famille que les choix iconographiques laissent hors champ. Grâce à la fonction référentielle de la photographie, qui se fait le vecteur de la présence humaine, les fictions relatées trouvent un ancrage dans l'univers évoqué, alors même qu'aucun personnage, au sens traditionnel (en pied, en mouvement), n’est montré.

\subsection{Objets métonymiques ou métaphoriques}

Les objets jouent le plus souvent un rôle métonymique. Dans Chaise et café, ce sont les deux termes du titre qui représentent les deux personnages, la chaise, grossièrement dessinée, comme par un enfant, et la petite tasse à café verte de l'adulte. Dans Chez Elle, l'image montre des lieux et des objets qui révèlent des personnes; ainsi, la personnalité frivole et narcissique d'une femme soucieuse de luxe et d'élégance est décrite, entre autres, par un défilé d'escarpins au premier plan, dans un appartement où se multiplient miroirs et attributs de la féminité.

L'objet peut aussi jouer un rôle métaphorique, comme l'ange de T'aurais tombé, qui représente manifestement l'enfant accidenté du récit, celui que ses parents appellent, non pas «notre ange », mais «notre roi », car il porte toujours une couronne de papier. Cet ange de plâtre, montré à l'ouverture et à la fermeture de l'album, et cassé lors de la chute de l'enfant, apparaît comme son «double symbolique ${ }^{9}$ ». A la dernière page, tête recollée, trônant au centre de la couronne, il semble sourire. C'est sans doute pour cela que Jean Perrot lui attribue la dernière phrase du texte : «Bonne nuit, majesté ! », où je vois plutôt les mots de la mère à l'enfant endormi.

\footnotetext{
${ }^{9}$ J. Perrot, Art baroque, art d'enfance, Presses Universitaires de Nancy, 1991, p. 269.
} 
Vêtements, objets du quotidien, accessoires divers, les objets parlent aussi, à leur façon, et saturent de traces humaines un univers qui paraissait désert. Certains ont un statut privilégié, puisqu'ils renvoient à des œuvres connues, dans tous les champs de la création, musique, peinture, sculpture, littérature. Dans cet univers, où clignotent mille signaux artistiques et culturels, arrêtons-nous un instant sur les livres, dont l'évocation relève aussi bien de l'intericonicité que de l'intertextualité.

\subsection{Livres}

Ce sont avant tout des livres pour enfants qui sont représentés, et parmi eux, des livres d'images. Accumulés, en piles, couchés ou debout, fermés ou ouverts, dessinés ou photographiés, tous ces livres dans le livre inscrivent l'album citant dans une filiation. Sur la couverture de T'aurais tombé, l'ouvrage que l'on s'apprête à ouvrir est cité de façon autoréférentielle, et disposé, en trompe l'œil, sur deux autres albums ; on peut reconnaître, en haut de la page, le titre de Cuisine de nuit, de Maurice Sendak ${ }^{10}$. Le livre intermédiaire à la couverture orange reste anonyme pour l'instant, mais il s'ouvrira un peu plus loin dans l'album et y tiendra son rôle : il s'agit de Babar en famille de Jean de Brunhoff, dans une version en noir et blanc, peut-être celle de 1938.

Les références culturelles de Poncelet la tournent vers les livres d'images « historiques » des XIXe et XXe siècles. A partir du loup en salopette de Philippe Corentin, on remonte le temps en croisant diverses figures du bestiaire plus ou moins anthropomorphisé de la littérature enfantine, l'Ours brun de Samivel, le Martin pêcheur de Rojankovsky, le Gédéon de Benjamin Rabier, mais aussi des classiques européens comme Max et Moritz et CrasseTignasse, traduction du Struwwelpeter par Cavanna. La référence littéraire passe parfois des livres aux objets de l'enfance, puisque les faces des cubes, dans l'album du même nom ${ }^{11}$, reprennent les images de Tenniel pour Alice au pays des merveilles et citent, de façon très éclectique, des contes de Perrault et des frères Grimm (Le Petit Poucet, Les musiciens de la ville de Brême) et même - on ne peut donc taxer Poncelet d'élitisme - des dessins animés de Walt Disney : on reconnaît Mickey, Pinocchio, Pan-pan le lapin de Bambi.

Ces références culturelles sont toujours motivées par l'atmosphère à peindre ou le drame qui se joue. Le «palimpseste pictural » devient ainsi, d'après Jean Perrot, « une technique de

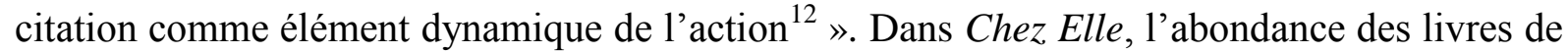
jeunesse témoigne de l'attachement d'un adulte au monde de l'enfance, et du désir de partager

\footnotetext{
${ }^{10}$ Le trompe l'œil se poursuit sur toute la couverture puisque la quatrième reprend celle de Cuisine de nuit.

${ }^{11}$ Sur l'intertextualité dans cet album, lire l'article de B. Gromer, «Les Cubes, de Béatrice Poncelet », Revue des Livres pour Enfants, n $^{\circ} 240$, mai 2008, p. 119-128.

${ }^{12}$ J. Perrot, op.cit., 279.
} 
livres et jeux avec la narratrice. La convocation des gravures de Doré illustrant les Contes de Perrault est toujours au service du propos : le loup du Petit Chaperon rouge surgit dans Je, le loup et moi, qui est une réécriture du conte ${ }^{13}$; dans Les Cubes, l'image récurrente de l'ogre égorgeant ses filles, d'autant plus saisissante que grossie et colorée, révèle l'inquiétude des enfants face à une aïeule qui se comporte étrangement, au point de les effrayer. Poncelet fait appel aux histoires en images qui figurent dans les strates de la mémoire collective pour traduire un sentiment, une émotion, comme dans T'aurais tombé, où la chute d'Alexandre dans Babar en famille est mise en relation avec celle de l'enfant accidenté du récit. Les références parcourent la gamme des âges, de l'imagier et de l'abécédaire au roman. La présence de 20000 lieues sous les mers dans Chut! elle lit, met en parallèle épopée maritime et soirée familiale ponctuée de rebondissements. Dans Les Cubes, les faces des cubes disséminées sur la page montrent des fragments d'images et traduisent l'impossibilité de recomposer l'image source, traduction visuelle de l'éclatement, du morcellement affectant une mémoire malade qui ne parvient plus à rassembler les bribes du réel. L'impossible reconfiguration de l'image des cubes est mise ici en parallèle avec le traitement des pixels de l'image numérique qui, lacunaires et disséminés, empêchent l'identification d'un portrait.

Mais tous les livres montrés ne relèvent pas de la littérature de jeunesse. Sur la première double page des Cubes, la photographie d'un ouvrage ouvert, et l'échange entre les deux narratrices, mettent en abyme le récit qui va suivre :

«... au fait, tu lis quoi ? ... MOI? LE LIVRE D’UN ECRIVAIN JAPONAIS, TRADUIT COMME TU VOIS... et ça raconte quoi ?... COMMENT ON S'HABITUE A VIVRE AVEC QUELQU'UN QUI CHANGE, DISONS... eh ben ! ! dis-donc !! pas très rigolo ton histoire !! »

Le sujet est ainsi annoncé, et même, sur l'image, l'une des péripéties du récit : la page montrée, au début du chapitre trois, relate un épisode repris dans l'album, celui où la mère du narrateur a perdu son sac, qu'on retrouve dissimulé sous une azalée. Le titre du livre est indiqué à la fin : il s'agit du récit autobiographique de Yasushi Inoué, Histoire de ma mère. La citation permet de mettre un nom sur le lien qui unit l'une des narratrices au personnage en souffrance, et d'aborder un sujet douloureux avec à la fois précision et distance.

\footnotetext{
${ }^{13}$ Je me permets de renvoyer à un article où j'analyse cet album : «Echos et chocs entre texte et images pour revisiter les Contes de Perrault», publié dans A l'œil. Des interférences textes/images en littérature, dir. J-P Montier, Presses Universitaires de Rennes, 2007, p. 27-36.
} 


\section{La prise en compte du lecteur}

On sait bien, depuis les travaux d'Umberto Eco, que le lecteur est inscrit dans la forme même du message, et aussi que ce lecteur peut être double, l'un davantage doté de «malice inférentielle et d'étendue encyclopédique », à moins que le lecteur naïf ne «grandi [sse] en astuce à la seconde lecture ${ }^{14} \gg$. Poncelet s'adresse manifestement à ce double lecteur modèle où l'on peut voir le couple formé par l'adulte et l'enfant.

\section{Plusieurs niveaux de temporalité}

Les voix qui s'expriment parlent au présent : présent de l'énonciation, et de l'adresse au lecteur. C'est à partir de cette temporalité particulière, le temps vécu par la conscience, celui dont elle fait l'expérience, que s'ouvrent des échappées, soit vers le passé, sous la forme d'un récit rétrospectif (souvenir de l'accident dans T'aurais tombé, récit de la rencontre dans Je, le loup et moi, description de la relation à l'autre dans Chaise et café), soit vers le futur, et porteuses d'hypothèses (inquiétude devant le vieillissement dans Et la gelée, interrogation sur l'impact des influences reçues dans Chez Elle). Ce sont les cas les plus «classiques ». Ces albums s'inscrivent dans une durée mesurable, un «temps chronologique ${ }^{15}$ » qui correspond au temps de la lecture : de $8 \mathrm{~h}$ à $8 \mathrm{~h} 23$ dit la voix didascalique de T'aurais tombé, album scandé par des apparitions d'horloges sur l'image; durée du petit déjeuner dans Et la gelée qui s'ouvre aussi sur une horloge ; on peut dire que les épisodes de Chut! elle lit se déroulent en temps réel sous les yeux du lecteur. Il existe donc toujours un niveau premier, facile à suivre, caractérisé par unité de lieu et de temps, tandis qu'à l'arrière-plan se creusent diverses incursions temporelles. Mais certains albums proposent une approche plus complexe de la temporalité. Leur format même, toujours un peu trop allongé, donne une traduction matérielle de la distance parcourue ou de la profondeur évoquée.

L'armature des Cubes, c'est ce journal tenu sur un carnet par la narratrice adulte, et reproduit, avec son écriture manuscrite, au centre de la première double page, entre le dialogue dans lequel il s'enchâsse et le récit d'Inoué, trois strates d'un palimpseste. Pendant un mois, ce texte manuscrit, aux allures de document, enregistre au jour le jour les progrès du drame, tissés dans la relation des activités quotidiennes et des nouvelles du monde. Parallèlement, on peut suivre le récit rétrospectif de la jeune narratrice, texte imprimé, présenté en pavé, récit plus conventionnel à entendre et à voir, plus organisé aussi, puisqu'il

\footnotetext{
${ }^{14}$ U. Eco, Lector in fabula, Grasset, 1979, p. 153.

${ }^{15}$ P. Ricœur, Temps et récit II, Seuil, 1984, p. 199.
} 
est le fruit d'une élaboration de la mémoire, et trie les souvenirs pour sélectionner les épisodes marquants de l'été précédent. Au contraire, dans le morcellement du carnet, tout se mêle, dans la captation immédiate de l'instant écoulé : considérations sur la nature, sur la vie quotidienne, et passim, indices inquiétants dans le comportement de l'aïeule.

Une autre configuration met en parallèle deux temporalités différentes, dans Semer en ligne ou à la volée, album particulièrement composite, à la fois herbier et guide de jardinage, succession de poèmes et d'aquarelles sur les états d'un jardin, au fil des saisons, où se glissent quelques haïkus, transcription d'épiphanies. Une seule narratrice ici, une femme qui jardine, tout en regardant grandir son enfant. On peut noter une distorsion temporelle entre les deux itinéraires parcourus simultanément. Au premier plan, le plus visible, la succession des saisons est nettement marquée sur l'image, en particulier par le jeu des couleurs. Une année s'écoule, d'un hiver à l'autre. Dans le même temps, semble-t-il, l'enfant, sous le regard maternel, fait ses premiers pas, joue ; puis ses jeux évoluent, se complexifient ; des outils et des instruments de mesure sophistiqués remplacent peu à peu mécano et lego ; à la fin de l'album, l'enfant est devenu un adulte. La métaphore du jardinage permet à Poncelet de suggérer aussi la croissance et l'éducation d'un enfant. Douze mois et trois décennies se déroulent ainsi en parallèle, à travers la voix de la narratrice, dont le regard subjectif voit toujours l'enfant dans l'homme, comme en atteste la photographie récurrente du premier, trace et reflet d'un instant unique, et d'un éternel présent. La succession des pages et des images rend palpable le cours du temps, temps objectif du cours des saisons, temps subjectif, affectif, élastique, du flux de conscience de la narratrice ; temps cyclique de la nature, temps linéaire de la vie humaine, les deux trajectoires se rejoignant lorsque la narratrice découvre, au dénouement, que l'enfant, devenu adulte, se met au jardinage à son tour, ce qui transforme le cours irréversible de la vie en un temps cyclique, lui aussi : jardinage métaphorique, s'entend, puisque la dernière image reprend les anneaux rose et bleu qui indiquaient, au début de l'album, l'âge de l'enfant, et suggèrent ici l'arrivée d'un nouvel enfant, chez le jeune adulte. Ainsi, l'ouvrage invite à une «expérience fictive du temps», selon le mot de Ricœur $^{16}$, expérience qui s'effectue à deux niveaux, à deux vitesses, peut-être pour deux niveaux de lecture différents.

Il faudrait aussi évoquer la manière dont les rêveries de la narratrice de Et la gelée lui font parcourir toute une vie, de la préadolescence aux vers du tombeau, pendant la durée du

\footnotetext{
${ }^{16}$ Ibid., p. 165.
} 
petit déjeuner, tandis que, comme le narrateur proustien qui voit surgir tout Combray d'une tasse de thé, elle contemple des pans entiers de l'histoire de l'art sortis de son bol de lait...

\section{Guidage par la voix}

Voix et images subjectives sont orientées vers le lecteur : le présent du texte renvoie au présent de la lecture et à la « mise en présence par l'image ».

Dans les albums les plus anciens, la voix narratrice s'adresse volontiers à un narrataire extradiégétique qui matérialise dans le texte la présence du lecteur par l'intermédiaire d'un «tu » invité à diriger son regard vers les détails de l'image, procédé courant dans les livres pour enfants ${ }^{17}$. Au début de Je pars à la guerre, je serai là pour le goûter, la phrase « Tu as vu ces plumes? Je les ai gardées exprès pour te les montrer », attire l'attention sur un indice qui ne prendra sens que plus loin. Le narrataire devient ainsi « un personnage actif du texte, un relais efficace entre la narration et le lecteur ${ }^{18}$ » pour orienter et baliser la lecture : «C'est la réception même du texte qui est mise en scène, décrite, $[\ldots]$ prévue $»^{19}$. La présence du narrataire contribue à l'animation et au rythme du discours narratorial, jalonné d'interpellations, d'impératifs, de questions, d'exclamations. La voix narrative qui invite à regarder l'image, relaie la voix de l'adulte qui lit le livre à l'enfant et le précède dans l'examen des doubles pages, lui montrant tel ou tel indice signifiant. Dans T'aurais tombé, lorsque la mère dit : «La valise on l'a retrouvée quand tu es tombé, ouverte comme ça, à côté de toi. Ensemble ils tournent la page. $\underline{\mathrm{Tu}}^{20}$ vois ?», le déictique invite simultanément personnage et lecteur à regarder l'image. On observe ici une de ces «infractions étranges à [la] séparation entre l'univers de la fiction et le hors-texte » dont parle Nathalie PiégayGros $^{21}$. Le récit fait apparaître un double destinataire, intra et extradiégétique, l'enfant de l'histoire et le lecteur. Après Galipette (1992), cet artifice de la convocation explicite du narrataire disparaît, et la relation entre texte et image s'opère sans son intermédiaire.

La voix est relayée par l'image dont la fonction conative s'exerce à travers les regards qui se plantent dans celui du lecteur : regard des photographies, toujours frontales, comme celle de la lolita qui pose devant l'objectif dans Je, le loup et moi ; regard de la narratrice de Et la gelée qui croise celui du lecteur à chaque page ; regards jumeaux des deux sœurs épiant leur mère dans Chut! elle lit. Même lorsqu'elle cite des images connues, Poncelet réoriente

\footnotetext{
${ }^{17}$ Comme dans Macao et Cosmage, ou dans le Struwwelpeter qui commence par «As-tu vu comme il est laid ?»

${ }^{18}$ N. Piégay-Gros, Le lecteur, GF Flammarion, 2002, p. 144.

${ }^{19}$ Ibid., p. 143.

${ }^{20} \mathrm{C}$ 'est moi qui souligne.

${ }^{21}$ Op. cit., p. 240.
} 
leur regard, comme on le voit avec le portrait de l'Européenne du Fayoum qui regarde le lecteur en face. On pourrait dire que l'œil est dans l'album et regarde le lecteur.

\section{Guidage iconique}

L'agencement des images prend fermement en charge le guidage du jeune lecteur, en procédant par enchaînements à la fois graphiques et sémantiques, anticipations et reprises. La lecture apparaît comme un itinéraire balisé au premier plan par des objets du quotidien, aisément reconnaissables, souvent en taille réelle, sorte d' « objets transitionnels » reliant le monde du lecteur et le monde en deux dimensions du livre : dans T'aurais tombé, une bande Velpeau se déroule sur 9 pages, et donne une cohésion plastique à la séquence narrative de l'accident ; dans Et la gelée, les objets du petit déjeuner occupent le premier plan, dans Semer, il s'agit des jouets et des outils de jardinage ; dans Chaise et café, la profondeur de champ de l'image distingue les univers représentés, celui de l'enfant au premier plan, celui de l'adulte à l'arrière. L'album qui paraît le plus inaccessible aux enfants, en raison de son sujet et de sa complexité graphique, Les Cubes, est aussi un abécédaire, qui comporte un premier niveau de lecture très facile à suivre : il suffit de connaître l'alphabet, qui s'égrène sur les faces des cubes, page après page (et même si le $\mathrm{W}$ intervient deux fois, comme indice du dérèglement observé dans le personnage de l'aïeule). Les cubes du titre et de l'image apparaissent à la fois comme jeu d'enfant et symbole de la maladie.

L'image obéit à une linéarité qui est celle du texte, et du temps. La succession des pages implique un travail constructif de mise en relation et en donne le mode d'emploi. Suivre les objets de premier plan dans Semer donne des indications sur la croissance de l'enfant évoqué. D'autre part, le début et la fin des albums sont systématiquement mis en relation. On sait que Poncelet compose ensemble toute la succession des pages, sur un chemin de papier de 16 mètres, afin de concerter le passage de l'une à l'autre et les échos entre elles. D'où ce jeu de prolepses, ces objets orientés vers la droite pour indiquer le sens de la lecture. D'où une construction circulaire où page d'ouverture et de clôture se répondent en construisant un cadre temporel et spatial : les masques de Je, le loup et moi, le livre, l'horloge et l'ange de T'aurais tombé, la boîte de cubes que l'on ouvre puis referme, le roman de Jules Verne, du premier chapitre au dernier dans Chut! elle lit... Notons que les albums se terminent toujours par une image placée sur la page de gauche, pour interrompre l'élan de la lecture, signifier et indiquer plastiquement la fin de l'ouvrage, en montrant porte, livre ou carton à dessin qui se referme. Même s'il n'est pas tout à fait achevé puisque Poncelet ajoute souvent un détail final, mot ou 
image, une phrase qui s'exclame, questionne ou reste en suspens, un détail, qui font rebondir l'intérêt et ouvrent de nouvelles perspectives ${ }^{22}$.

Ce qui paraissait problématique à première vue est en réalité construit dans un rapport constant au jeune lecteur, et toutes ces balises lui permettront de relire seul le livre qui lui a été lu par l'adulte. Dans ce magasin de curiosités où les objets s'accumulent, ce labyrinthe apparemment désert, où s'ouvrent des portes, des voies où l'on peut s'égarer, où certains chemins sont réservés au seul lecteur adulte, on découvre une composition très concertée, faite d'échos et de symétries. De plus, reliant un album à l'autre, la récurrence de motifs privilégiés concourt à la cohérence de cet univers : jouets (la sauterelle, la toupie), objets (les bols à pois), œuvres d'art (Doré, Modigliani, le portrait du Fayoum). L'œuvre est parsemée de fils conducteurs, de panneaux indicateurs, et le livre prétendument hermétique comporte une notice de montage.

Ces albums singuliers, je les qualifierais volontiers ${ }^{23}$ de «livres d'artiste » en me fondant sur la phrase d'Edward Ruscha : «Je n'essaie pas de créer un livre précieux en édition limitée, mais un produit de série qui soit de premier ordre », citée par Anne MoeglinDelcroix, qui définit le livre d'artiste comme « un livre entièrement conçu par un artiste, à tirage non limité, et utilisant les moyens ordinaires du livre ${ }^{24} »$. En effet, Béatrice Poncelet assume toute la fabrication de ses ouvrages, de la conception jusqu'à la réalisation matérielle du produit fini, du chemin de papier initial déroulé dans son atelier, jusqu'à la maquette réalisée entièrement à la main qu'elle présente à l'éditeur, puis au suivi de l'impression chez l'imprimeur, où, bien qu'elle n'aille pas jusqu'à actionner les rotatives, elle veille à la fidélité de la reproduction. Dans ces ouvrages extrêmement personnels, exempts de tout paratexte éditorial comme de pages de titre, elle décide de tout, jusqu'à la place réservée au code barres ou au copyright.

Plusieurs d'entre eux, T'aurais tombé, Chez Elle, Chut! elle lit, et le dernier paru, Le Panier, l'immense panier, évoquent la lecture d'un adulte et d'un enfant dans le cadre familial, scène où Denise von Stockar voit la représentation d' « une déclaration d'amour à la

\footnotetext{
22 I.Nières mentionne les albums de Poncelet lorsqu'elle analyse les différents types de «clausules iconographiques» dans son article «Et l'image me fait signe que le livre est fini », Culture, texte et jeune lecteur, dir. J. Perrot, Presses universitaires de Nancy, 1993, p. 209-217.

${ }^{23}$ Toujours contre la modestie d'auteur de B. Poncelet.

${ }^{24}$ A. Moeglin-Delcroix, Sur le livre d'artiste. Articles et écrits de circonstances, 1991-2005, Le Mot et le reste, 2006, p. 104.
} 
lecture ${ }^{25} \gg$. C'est bien une lecture pour tous qui est proposée à travers ces albums singuliers, dont l'hermétisme apparent est un leurre. Abordables par tous, nourrissants pour tous, ces livres cultivés et émouvants invitent à une visite qui relève d'une expérience sensible dont je n'ai pu qu'effleurer la richesse ici.

Mais ces albums ne sont pas exclusivement réservés à la lecture dans le cercle familial ; ils se prêtent tout particulièrement, me semble-t-il, à la lecture littéraire à l'école, pour placer les enfants en situation problème, attirer l'attention sur les choix énonciatifs, les non-dits, car ils suscitent, par leur «résistance», une intense activité de lecture pour assembler un puzzle de signes savamment éparpillés. Leur densité proscrit le feuilletage superficiel et implique de multiples relectures, à l'affût d'indices textuels et surtout visuels, comme, par exemple, l'utilisation ludique de la typographie qui transfère les mots dans la sphère visuelle. Pour les lire à l'école, il faut surtout combattre une autre résistance, celle des médiateurs qui les classent dans la catégorie de ces albums contemporains complexes qui gratifient le chercheur, mais risquent d'exclure «le jeune public auquel ils prétendent s'adresser ${ }^{26} »$. Heureusement, l'un d'eux a été retenu sur une liste d'ouvrages recommandés par le Ministère de l'éducation nationale, ouvrant ainsi à tous la possibilité de vivre et de partager l'expérience et l'aventure de sa lecture.

Christiane Connan-Pintado

Bordeaux IV(IUFM)

TELEM, Modernités (EA 41-95)

\footnotetext{
${ }^{25}$ D. Von Stockar, «Le rôle de la musique dans Chut! elle lit de Béatrice Poncelet », dans Musiques du texte et de l'image, dir. Jean Perrot, CNDP, 1997, p. 203.

${ }^{26}$ A.Vibert, «A propos de Lire l'album de Sophie van der Linden », Texte et images dans l'album et la bande dessinée pour enfants, Les cahiers de Lire écrire à l'école, Scérén, CRDP, Académie de Grenoble, 2007, p. 169.
} 